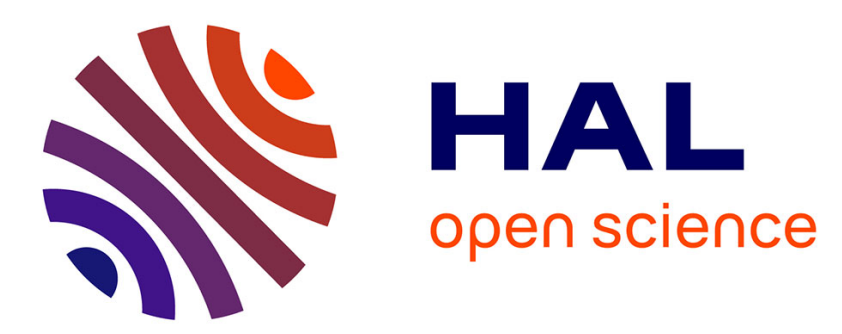

\title{
Direct numerical simulations of the shock-induced separation of a laminar boundary layer
}

Guillaume Fournier, Amer Chpoun, Yann Fraigneau, Christian Tenaud

\section{To cite this version:}

Guillaume Fournier, Amer Chpoun, Yann Fraigneau, Christian Tenaud. Direct numerical simulations of the shock-induced separation of a laminar boundary layer. Direct and Large-Eddy Simulation X, 24, pp.327-332, 2017, 9783319632117. 10.1007/978-3-319-63212-4_41. hal-01629644

\section{HAL Id: hal-01629644 \\ https://hal.science/hal-01629644}

Submitted on 18 Jul 2018

HAL is a multi-disciplinary open access archive for the deposit and dissemination of scientific research documents, whether they are published or not. The documents may come from teaching and research institutions in France or abroad, or from public or private research centers.
L'archive ouverte pluridisciplinaire HAL, est destinée au dépôt et à la diffusion de documents scientifiques de niveau recherche, publiés ou non, émanant des établissements d'enseignement et de recherche français ou étrangers, des laboratoires publics ou privés. 


\title{
Direct Numerical Simulations of the Shock-Induced Separation of a Laminar Boundary Layer
}

\author{
G. Fournier, A. Chpoun, Y. Fraigneau and C. Tenaud
}

\section{Introduction}

In the aeronautical and aerospace industries, the flow configurations where an incident oblique shock wave impinges upon a boundary layer are very common. The supersonic flow around an aircraft, in a rocket nozzle or in a supersonic inlet are some examples among others. Under certain circumstances (High Mach number, large shock angle ...), the interaction between the incident shock wave and the boundary layer may create an unsteady separation bubble. This separation bubble as well as the subsequent reflected shock that occurs around the separation point, are known to oscillate in a low-frequency streamwise motion that can spread over several tenth of the boundary layer thickness [12]. The origin of those oscillations, however still unclear, has been related either to the shedding of vortices in the mixing layer downstream of the separation, both for a flat-plate [2] or a ramp configuration [9], or to the turbulent structures in the incoming boundary layer [5]. Thanks to the tremendous increase of the computational power that has occured in the last two decades, many numerical studies of the shock wave/boundary layer interactions (SWBLI) have been performed recently using Large Eddy Simulations (LES) [1, 7, 14], RANS/LES hybrid methods [6] or Direct Numerical Simulations (DNS) [4, 15].

\footnotetext{
G. Fournier $(\varangle) \cdot$ A. Chpoun

Laboratoire de Mécanique et d'Energétique d'Evry, 40 Rue du Pelvoux,

91020 Evry Cedex, France

e-mail: guillaume.fournier@ufrst.univ-evry.fr
}

\author{
A. Chpoun \\ e-mail: a.chpoun@iut.univ-evry.fr \\ Y. Fraigneau $\cdot$ C. Tenaud \\ LIMSI-CNRS, Rue John von Neumann, \\ 91045 Orsay Cedex, France \\ e-mail: Yann@limsi.fr \\ C. Tenaud \\ e-mail: Christian.Tenaud@limsi.fr
}


Most of these studies however dealt with turbulent boundary layer and only a very few of them considered the laminar case $[10,11]$. The streamwise motion being potentially related to the turbulent behavior of the incoming boundary layer, the aim of the present study is therefore to show that the streamwise motion could also be recovered with an incoming laminar boundary layer. To investigate this streamwise motion, the interaction between a laminar boundary layer and an oblique shock has been studied using DNS.

\section{Numerical Methods}

In this study, Direct Numerical Simulations (DNS) of the interaction between an oblique shock wave and a laminar boundary layer have been performed using an in-house parallel (MPI) Finite-Volume based DNS/LES solver developed at LIMSICNRS [13]. A Monotonicity-Preserving shock-capturing scheme, based on the LaxWendroff method through a 7 th order accurate coupled space and time approximation is used for the convective fluxes [3]. The diffusive fluxes are discretized by a second order centered scheme.

The incoming laminar boundary layer is modelled using a 4th order polynomial approximation of the Blasius profile, defined by $\frac{u(z)}{U_{\infty}}=\frac{z}{\delta_{0}}\left(2-2\left(\frac{z}{\delta_{0}}\right)^{2}+\left(\frac{z}{\delta_{0}}\right)^{3}\right)$ where $z$ is the wall-normal direction and $\delta_{0}$ the boundary layer thickness. This function has the advantage to verify the boundary conditions both for the velocity at the wall and outside the boundary layer, and for the first-order derivative at $z=\delta_{0}$ and for $z \rightarrow \infty$. The Rankine-Hugoniot relations are used to create an oblique shock the inlet plane, which freestream Mach number and angle with respect to the horizontal plane fit the conditions from [8] and are respectively $M=2.23$ and $\beta=33.1^{\circ}$. Finally, an adiabatic no-slip condition is applied on the lower boundary at the solid wall. The Reynolds number based on the inlet boundary layer thickness is chosen to be $R e=1,800$ for the 2D case and $R e=1,000$ for the 3D simulations.

\section{Results}

2D simulations have first been conducted. The computational domain is discretised using $M=1000 \times 252$ cells and extends over $D=500 \delta_{0} \times 200 \delta_{0}$. In the wall-normal direction, a $3 \%$ geometrical stretching is applied in order to save CPU time without spoiling the space order of the scheme. An instantaneous field of the numerical schlieren is represented in Fig. 1 and clearly underlines the shock-induced separation of the laminar boundary layer and the subsequent recirculation bubble.

A qualitative comparison between these results and the reference studies shows that every aspects of such interactions are recovered. The incident shock, the reflected shock, the expansion fan and the reattachment shock are obvious, forming a very 


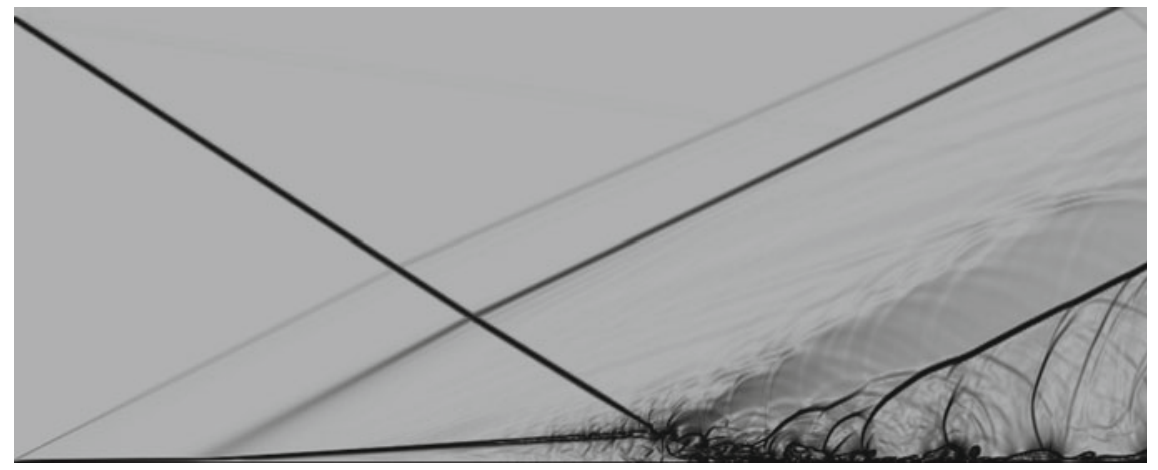

Fig. 1 Instantaneous contours of the numerical schlieren from a 2D simulations
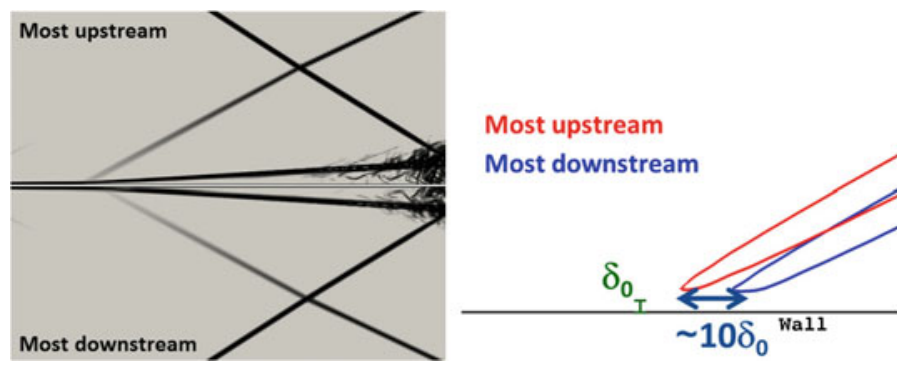

Fig. 2 Comparison of the most upstream and most downstream locations of the reflected shock, obtained for two different timesteps and revealed by contours of the numerical schlieren (left) and of the divergence of the velocity (right)

complex shock system. It is noteworthy that because of the laminar aspect of the incoming boundary layer, the extent of the recirculation zone is overestimated in the present simulations with respect to other numerical investigations, performed for a turbulent boundary layer. In addition, it can be seen in Fig. 2 that the reflected shock is submitted to an oscillatory motion that spreads over several $\delta$. This observation, though in qualitative agreement with results from [12], is also overestimated since Selig et. al. noted that the shock moved for only around one inlet boundary layer thickness. This quantitative discrepency could be partially explained by the laminar aspect of the boundary layer but some further investigations are in progress in order to conclude. However, these slight discrepencies should not minimise the fact that an oscillatory motion is found even with 2D simulations of a laminar boundary layer, that tends to prove that it is not solely due to the incoming turbulent structures.

$3 \mathrm{D}$ simulations have then be performed in order to show that the oscillatory motion that have been found in 2D simulations is not an artefact due to the lack of the third component. The analysis of the numerical schlieren (not shown in this paper) clearly shows that the reflected shock is subjected to a streamwise motion. However, no oscillations have been found and the reflected shock is just moving upstream. 


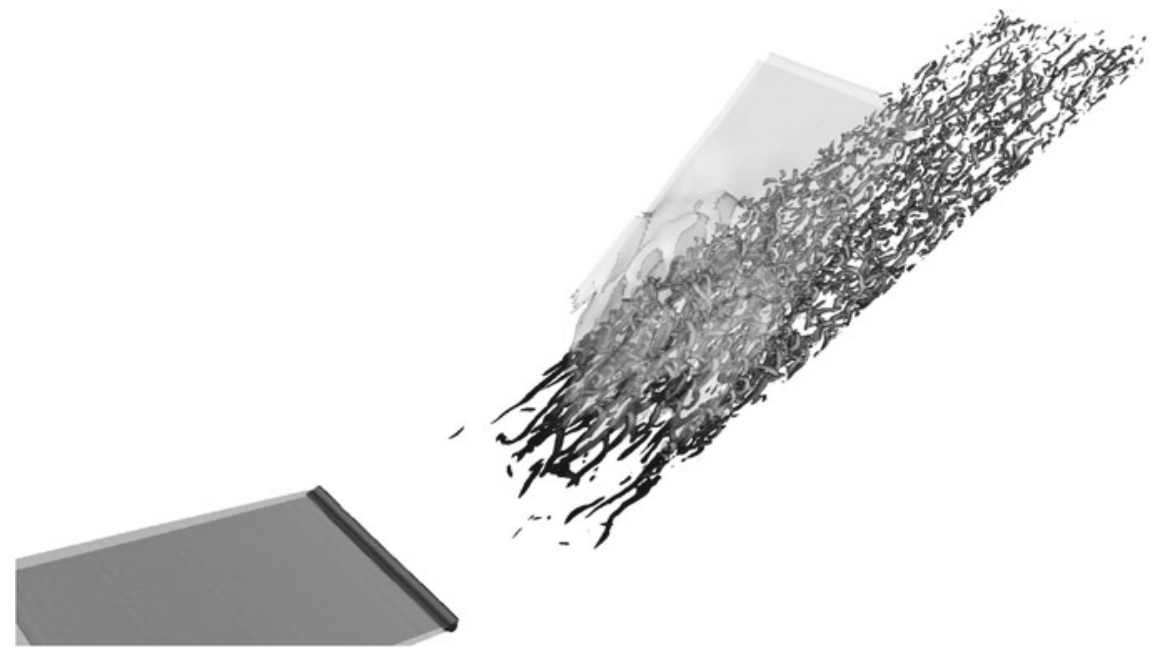

Fig. 3 Vortical structures in the interaction region, represented by Q criterion isosurfaces and coloured with the streamwise velocity (Low velocities are in black while the high velocities are in light gray). The shock system (translucent gray) is made visible by isosurfaces of the divergence of the velocity

The isosurfaces of the Q-criterion, coloured by the streamwise velocity are shown in Fig. 3. Isosurfaces of the divergence of the velocity are used to reveal the shock system (in light gray). Despite the 2D aspect of the inlet boundary layer, due to its laminar nature, a fully three-dimensional flow can be observed. The shock impinging the boundary layer leads to the creation of a highly three-dimensional separation bubble. The separation is first laminar and no vortical structures are observed. Then, the shear layer becomes unstable and the transition to turbulence occurs. This transition takes place just a few boundary layer thicknesses upstream of the reattachment point, as emphasised by the dark colour of the vortical structures, corresponding to low velocities. Once the boundary layer is reattached, they are elongated by the reacceleration occuring through the boundary layer and eventually evolve into large hairpin vortices, typical of wall-bounded turbulent flows.

This shedding is however very irregular. As shown in Fig. 4, the vortical structures are not uniformly shed over the span but it takes place at different locations at different timesteps, creating some spots with no structures. Those no-vorticity spots are clearly recovered on the time evolutions of the spanwise velocity for two probes, located in the vicinity of the reattachment point, at midspan and 20\% of the span (Fig. 5). At mid span, three occurences of these spots can be seen. Two very large spots can be found for $t \frac{U_{\infty}}{\delta_{0}} \in[0 ; 200]$ and $t \frac{U_{\infty}}{\delta_{0}} \in[800: 1000]$. In those cases, the spots seem to be limited to the midspan since the signal at the $20 \%$-span location does not exhibit any peculiar behaviour and therefore correspond to the configuration represented in Fig. 4-Left. On the other hand, the shedding cases of Figs. 4-Center and 4-Right are found either for $t \frac{U_{\infty}}{\delta_{0}} \sim 1100$ or $t \frac{U_{\infty}}{\delta_{0}} \sim 2500$. In addition to the 

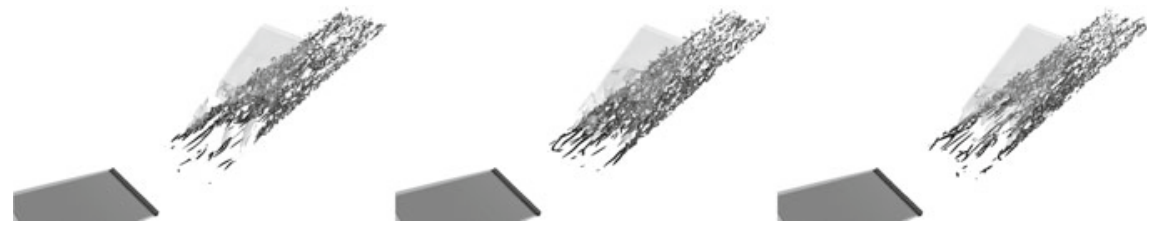

Fig. 4 Vortical structures for three different timesteps. Left Vortices shed only on the sides. Centeri Vortices shed only on the left part. Right Vortices only shed at midspan

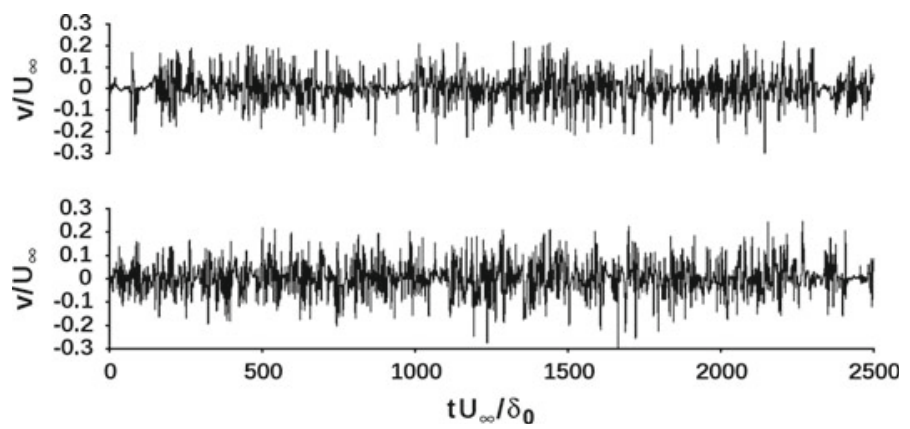

Fig. 5 Time history of the spanwise velocity for two probes located at midspan (Top) and at 20\% of the span (Bottom)

large shedding frequency, the signals exhibit at least two more frequencies. The first one corresponds to no-vortices spots passing around the probe while the other one is more likely related to the oscillations of the reattachment shock. Spectral analysis is currently in progress to precisely define those frequency and to find a more physical insight.

\section{Conclusions}

DNS have been performed in the case of the interaction between an oblique shock and a laminar boundary layer developing along a flat plate. The aim was to determine whether the oscillation motion of the separation bubble and the reflected shock is related to the turbulent aspect of inlet boundary layer or if it can happen even in for a laminar boundary layer. The preliminary 2D simulations have found that streamwise oscillations are recovered for the reflected shock. These promising preliminary results leaded us to perform 3D simulations. Up to date, no oscillations have yet been recovered with these simulations. The reflected shock has been shown to move upstream but no clear frequency has been found. Investigations are under way to see if the fact that the oscillations do not occur in the $3 \mathrm{D}$ case can be related to the different Reynolds numbers in 2D and 3D. The 3D simulations have however highlighted another interesting phenomenon. Even if the transition occurs in the 
shear layer just upstream of the reattachment point, the vortex shedding is not uniform after the boundary layer reattaches. Instead, some no-vorticity spots are created that highly increase the three-dimensionality of the flow and therefore increases the non-uniformity of the stresses.

Acknowledgements This work has been performed using HPC resources from GENCI-IDRIS, France (Grant 2015-i20152a7195).

\section{References}

1. Agostini, L., Larchevêque, L., Dupont, P., Debiève, J.-F., Dussauge, J.-P.: Zones of influence and shock motion in a shock/boundary-layer interaction. AIAA J. 50 (2012)

2. Aubard, G., Gloerfelt, X., Robinet, J.-C.: Large-Eddy simulation of broadband unsteadiness in a shock/boundary-layer interaction. AIAA J. 51 (2013)

3. Daru, V., Tenaud, C.: High order one-step monotonicity preserving schemes for unsteady flow calculations. J. Comp. Phys. 193 (2004)

4. Fang, J., Yao, Y., Li, Z., Lu, L.: Investigation of low-dissipation monotonicity-preserving scheme for direct numerical simulation of compressible turbulent flows. Comput. Fluids 104 (2014)

5. Ganapathisubramani, B., Clemens, N.T., Dolling, D.S.: Effects of upstream boundary layer on the unsteadiness of shock-induced separation. J. Fluid Mech. 585 (2007)

6. Garnier, E.: Stimulated detached eddy simulation of three-dimensional shock/boundary layer interaction. Shock Waves 19 (2009)

7. Grilli, M., Schmid, P. J., Hickel, S., Adams, N. A.: Analysis of unsteady behaviour in shockwave turbulent boundary layer interaction. J. Fluid Mech. 700 (2012)

8. Pirozzoli, S., Grasso, F.: Direct numerical simulation of impinging shock wave/turbulent boundary layer interaction at $\mathrm{M}=2.25$. Phys. Fluids 18 (2006)

9. Priebe, S., Martín, M. P.: Low-frequency unsteadiness in shock wave-turbulent boundary layer interaction. J. Fluid Mech. 699 (2012)

10. Robinet, J. C.: Bifurcations in shock-wave/laminar-boundary-layer interaction: global instability approach. J. Fluid Mech. 579 (2007)

11. Sansica, A., Sandham, N.D., Hu., Z.: Forced response of a laminar shock-induced separation bubble. Phys. Fluids 26 (2014)

12. Selig, M. S., Andreopoulos, J., Muck, K. C., Dussauge, J.-P., Smits, A. J.: Turbulence structure in a shock wave/turbulent boundary-layer interaction. AIAA J. 27 (1989)

13. Tenaud, C., Fraigneau, Y., Daru, V.: Numerical simulation of the turbulent separation reattachment flow around a thick flat plate. J. Phys. Conf. Ser. 318 (2011)

14. Touber, E., Sandham, N. D.: Large-eddy simulation of low-frequency unsteadiness in a turbulent shock-induced separation bubble. Theor. Comp. Fluid Dyn. 23 (2009)

15. Wu, M., Martín, M. P.: Analysis of shock motion in shockwave and turbulent boundary layer interaction using direct numerical simulation data. J. Fluid Mech. 594 (2008) 